Arthur André Leto Barreto

Estudo do rendimento térmico de motores propulsores de dois tempos operando em regime transiente

Dissertação de Mestrado

Dissertação apresentada como requisito parcial para obtenção do título de Mestre pelo Programa de PósGraduação em Engenharia Mecânica da PUC-Rio.

Orientador: Luís Fernando Figueira da Silva 


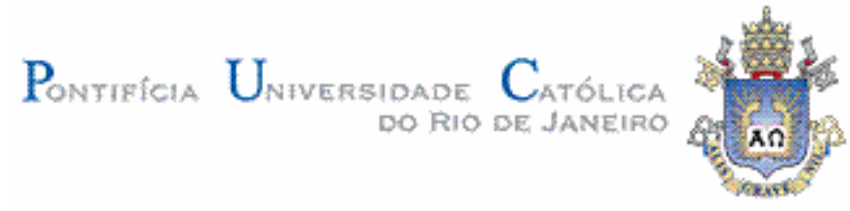

Arthur Andre Leto Barreto

\section{Estudo do rendimento térmico em motores propulsores de dois tempos operando em regime transiente}

Dissertação apresentada como requisito parcial para obtenção do título de Mestre pelo Programa de Pós-Graduação em Engenharia Mecânica da PUC-Rio. Aprovada pela Comissão Examinadora abaixo assinada.

Prof. Luís Fernando Figueira da Silva Orientador Departamento de Engenharia Mecânica - PUC -Rio

Prof. Sergio Leal Braga Departamento de Engenharia Mecânica -PUC-Rio

Dr. Sandro Barros Ferreira Instituto de Energia - PUC -Rio

Prof. José Eugênio Leal Coordenador(a) Setorial do Centro Técnico Científico - PUC-Rio 
Todos os direitos reservados. É proibida a reprodução total ou parcial do trabalho sem autorização da universidade, do autor e do orientador.

\section{Arthur André Leto Barreto}

Engenheiro Mecânico graduado na Universidade Gama Filho em 1980. Realiza trabalhos de projeto de plantas, seleção e manutenção de motores marítimos. Consultor Técnico da Petróleo Brasileiro S.A, na área de combustíveis residuais, lubrificantes e motores marítimos.

Ficha Catalográfica

Barreto, Arthur André Leto

Estudo do rendimento térmico em motores propulsores de dois tempos operando em regime transiente/ Arthur André Leto Barreto; orientador: Luís Fernando Figueira da Silva. Rio de Janeiro: PUC, Departamento de Engenharia Mecânica, 2009.

102 p.:il.; $30,0 \mathrm{~cm}$

1. Dissertação (Mestrado) - Pontifícia Universidade Católica do Rio de Janeiro, Departamento de Engenharia Mecânica.

Inclui referências bibliográficas.

1. Engenharia mecânica - Teses. 2. Motores de dois tempos-controle. 3. Rendimento Térmico. 4. Motores Propulsores.5. Funções de transferência. 6. Modelagem. 7. Torque transiente. I.Figueira da Silva, Luís Fernando. II. Pontifícia Universidade Católica do Rio de Janeiro, Departamento de Engenharia Mecânica. III. Título. 


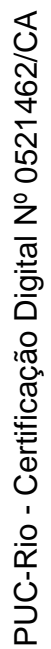

Dedicado à minha esposa Patrícia e aos meus filhos Antônio, Tarso e Caio. 


\section{Agradecimentos}

Ao meu orientador Professor Luís Fernando Figueira da Silva, pela confiança e estímulo na realização desse trabalho.

Aos meus professores e colegas da PUC Rio.

À Petrobras-Transpetro, por proporcionar essa oportunidade de crescimento pessoal e profissional.

À minha família pela paciência e colaboração.

Aos professores da Comissão Examinadora. 


\section{Resumo}

Barreto, Arthur André Leto; Silva, Luís Fernando Figueira da (orientador). Estudo do rendimento térmico em motores propulsores de dois tempos operando em regime transiente. Rio de janeiro, 2009. 102p. Dissertação de Mestrado - Departamento de Engenharia Mecânica, Pontifícia Universidade Católica do Rio de Janeiro.

Atualmente o motor diesel é a propulsão básica utilizada por navios mercantes. Predominantemente o arranjo mais utilizado é o de um motor de dois tempos turboalimentado diretamente acoplado ao hélice. Estes motores são controlados por um regulador de velocidade onde um valor desejado da rotação é ajustado. O regulador opera acionando as réguas das bombas injetoras, movendo-as conforme a necessidade de manter a rotação desejada mesmo sob torque transiente. Se o rendimento térmico é reduzido, os índices das bombas injetoras terão que operar em valores mais altos, quando comparado ao funcionamento do motor com o rendimento térmico de projeto, e neste caso maior geração de fuligem deverá ocorrer. Em motores de combustão interna, a deposição de fuligem trás consequências à manutenção e eficiência do motor, e por isto os operadores possuem motivos para evitar este tipo de mecanismo. Este trabalho propõe um modelo termodinâmico dotado de sub modelo para regulador de velocidade e para a fluturação do torque, o que permite observar o rendimento térmico nas condições de transiência. Dados adquiridos por um sistema de aquisição de dados fornece as condições de contorno para ajustar o modelo proposto.

\section{Palavras-chave}

Motores de dois tempos - Controle, Rendimento Térmico, Motores Propulsores, Funções de Transferência, Modelagem, Torque Transiente. 


\section{Abstract}

Barreto, Arthur Andre Leto. Silva, Luís Fernando Figueira da (Advisor).Simulation of thermal efficiency at two strokes propulsion engines under transient torque. Rio de janeiro, 2009. 102p. MSc. Dissertation- Departamento de Engenharia Mecânica, Pontifícia Universidade Católica do Rio de Janeiro.

The marine diesel engine is today's predominant prime mover for ships propulsion. For the modern merchant vessels, the arrangement of a single slow speed turbocharged two stroke diesel engine directly coupled is used. The task of control these engines are done by speed governors, where a fixed rotational speed is set. The governor works holding the adjusted value even under transient loads, moving the fuel pump rack when necessary. When vessel operates in harsh weather conditions, the propellers may be subjected to large thrust and torque fluctuations. These fluctuations are generated by the propeller periodic change of submergence condition which leads to ventilation (air suction) and partial or full propeller emergence, at same time the rudder action to keep the route introduce another torque fluctuation. Thus, propulsion turbocharged two strokes engines runs under transient load could run with low fuel/air ratio, once the turbocharger can not be able to cover this transient range with appropriate response time. Some models from literature, like control and thermodynamics models are examined and adjusted with sub models for transient torque simulation. One thermodynamic model dotted of sub models for governor, and transient load is proposed and the thermal efficiency under transient torque observed. Data from monitored vessel's engine under operation, supplied the boundaries conditions to adjust the proposed model.

\section{Keywords}

Two stroke engines - Control, Thermal Efficiency, Propulsion Engines, Transfer Function, Models and Transient Torque. 


\section{Sumário}

1 . Introdução

2. Modelagem de ciclo motor em regime permanente 23

2.1. Ciclo motor: modelagem clássica. 23

2.1.1. Descrição do ciclo motor a ar 23

2.2. Ciclo turbo alimentado 32

2.3. Turboalimentador 34

2.4. Relação entre motor e hélice do navio. 36

2.5. Diagrama de Carga 36

3. Modelos de funcionamento transiente de motores a dois tempos. 40

3.1. Função de transferência dotada de regulador de velocidade PI(D). $\quad 40$

4. Modelos quase estáticos de motores de dois tempos. 50

4.1. Combustão em modelos quase estático 50

4.2. Resultados obtidos.

4.2.1. Resultados obtidos- Modelo quase estático Clássico 57

4.2.2. Resultados do Modelo modificado.

4.3. Elaboração de modelo quase estático dotado de regulador 61

4.3.1. Descrição do modelo. $\quad 61$

4.3.2. Resultados obtidos 68

5. Resultados experimentais obtidos a bordo 76

5.1. Descrição do experimento 76

5.2. Análise dos resultados experimentais. 78

5.2.1. Descrição dos dados como obtidos e seu tratamento. 79

6. Comparação entre os resultados experimentais e de modelagem 82

6.1. Resultados comparativos 82 
6.2. Análise do rendimento térmico 84

6.3. Análise do comportamento transiente 86

7. Conclusões e perspectivas $\quad 89$

8. Referências bibliográficas 92

9. APÊNDICE I 95 


\section{Lista de figuras}

Figura 1- Representação de um motor propulsor de dois tempos. 24

Figura 2- Diagrama pV do ciclo motor ar teórico misto. 26

Figura 3- Diagrama $\mathrm{pV}$ fechado e aberto de um motor real obtidos de um motor propulsor de dois tempos.

Figura 4- Representação do ciclo real fechado e aberto com os pontos significativos do ciclo teórico. 28

Figura 5- Ciclo misto com turboalimentador à pressão constante. 33

Figura 6- Mapa de demanda de potência, casco, hélice e motor para um determinado navio.

Figura 7- Diagrama de bloco do simulador do motor dotado de regulador de velocidade, e função de transferência.

Figura 8- Diagrama de blocos do regulador $\mathrm{PI}(\mathrm{D})$ utilizado no controle do FPI.

Figura 9- Histórico do índice das bombas injetoras determinado pelo modelo encontrado na literatura (Kyrtatos et al., 2001) (superior), e o resultado da implementação no modelo da Figura 7.

Figura 10- Evolução da rotação $(\mathrm{Ne})$ da simulação do modelo descrito em Kyrtatos et al., (2001).

Figura 11- Evolução do índice das bombas injetoras sob a correspondente redução do rendimento térmico.

Figura 12- Evolução da reação do sistema a um sinal degrau de 100 kN.m, no instante de $300 \mathrm{~s}$, e um sinal de torque transiente contínuo de $200 \mathrm{kN} . \mathrm{m}$, frequência de $0,624 \mathrm{rad} / \mathrm{s}$. O ganho termodinâmico de projeto foi reduzido em $5 \%$..

Figura 13-Evolução da resposta de rotação do modelo encontrado na literatura (Kyrtatos et al, 2001) nas condições de rendimento térmico original e reduzido em $5 \%$.

Figura 14-Desenho esquemático de motor de dois tempos para modelagem sob torque estático e transiente, Hendricks (1989).

Figura 15-Representação da área equivalente às áreas de abertura 
de válvula de descarga e janelas de admissão, Meier (1981).

Figura 16-Proporção da energia química do combustível no gás de descarga, $\zeta_{a}$, como função da pressão média efetiva $p_{e}$. Para motores dois tempos (a), e motores de quatro tempos $(b)$, Meier(1981).

Figura 17-Histórico da rotação do turbo compressor e do motor obtida para um motor de $11 \mathrm{MW}$ conforme encontrado na literatura (Xiros, 2002)

Figura 18-Histórico das simulações da rotação do turbo compressor e do motor de $11 \mathrm{MW}$ (Xiros, 2002), calculada pelo modelo algébrico quase estático acrescido de função entre FPI e a pressão de ar de alimentação.

Figura 19-Histórico da variação do rendimento térmico e da razão ar combustível no motor de $11 \mathrm{MW}$, extensão ao modelo de Xiros (2002).

Figura 20-Histórico da variação de rotação do motor de 17 MW.

Figura 21-Simulação da temperatura de gás de descarga antes do turbo para o motor de $17 \mathrm{MW}$.

Figura 22-Diagrama de bloco do modelo completo, formado por diversos sub modelos, descritos separadamente.

Figura 23 - Diagrama de bloco do sub sistema de determinação da aceleração do turbo compressor.

Figura 24-Sub sistema do diagrama de blocos do modelo para torque no hélice com perturbação.

Figura 25-Representação do diagrama de bloco das funções do modelo para o cálculo da aceleração do motor e da linha de eixo.

Figura 26-Sub modelo para Cálculo do rendimento térmico.

Figura 27-Representação do sub modelo de comando e regulagem.

As constantes de proporcionalidade e integralidade são aquelas sugeridas por Kyrtatos et al., (2001).

Figura 28-Histórico do cmportamenteo do rendimento térmico do motor de $17 \mathrm{MW}$, rendimento térmico original é alterado pela redução de $5 \%$ da pressão média efetiva.

Figura 29-Histórico da evolução do sinal de rotação do motor no modelo. A evolução do sinal com o modelo com rendimento térmico reduzido e de projeto encontram-se superpostos. 
Figura 30-Histórico dos sinais de rotação do motor e dos sinais de índice das bombas injetoras.

Figura 31-Histórico do resultado de simulação da rotação do turbo compressor do modelo de motor de $17 \mathrm{MW}$, nas condições de rendimento térmico de projeto e reduzido.

Figura 32-Histórico da evolução da temperatura de gás de descarga na entrada do turbo compressor, modelo com sinal de torque transiente cíclico, nas condições de rendimento termico de projeto e reduzido.

Figura 33-Histórico da razão ar/combustível no modelo com rendimento térmico reduzido e com o rendimento térmico de projeto.

Figura 34-Histórico do consumo diário do motor com o rendimento térmico de projeto e com o rendimento térmico reduzido.

Figura 35-Esquema de colocação de sensores de aquisição de dados.

Figura 36-Histórico da velocidade de rotação do motor. Resultados experimentais brutos, e a senoide correspondente.

Figura 37-Histórico dos dados e curva correspondente de rotação do turbo.

Figura 38-Histórico da medição do sinal do índice das bombas injetoras

Figura 39-Histórico da pressão do ar de alimentação entre a montante e a jusante do resfriador de ar de lavagem.

Figura 40-Históricos da rotação do motor, calculado e medido.

Figura 41-Comparação entre os históricos entre a pressão de ar alimentação calculada e a pressão de ar de alimentação medida a bordo.

Figura 42-Aspecto da superposição do desenvolvimento da rotação do turbo medido e calculado.

Figura 43-Descrição do consumo diário de combustível (linha tracejada) e rendimento térmico conforme a redução da pressão média efetiva.

Figura 44-Resultado na termperatura de gás de descarga (superior) e no índice das bombas injetoras (inferior) devido à redução na pressão média efetiva.

Figura 45-Histórico da velocidade de rotação do motor entre as condições de rendimento térmico degradado em $10 \%(\mathrm{Ne} 1)$ e original $(\mathrm{Ne})$. 
Figura 46-Histórico da velocidade de rotação do turbo compressor e do índice das bombas injetoras com o modelo ajustado com rendimento térmico original (Nt, FPI*100) e degradado (Nt100, FPI10*100).

Figura 47-Histórico da temperatura de gás de descarga na entrada do turbo com rendimento térmico degradado em $10 \%$ (Tg1) e o original (Tg).

Figura 48-Polinômio representando a equação 4.13, que deduz a pressão de saída do compressor em $m m H g . R^{\wedge} 2=1$.

Figura 49-Polinômio representando a equação 4.14, que deduz a temperatura de gás após a turbina. $R^{\wedge} 2=1$.

Figura 50-Polinômio que representa a equação 4.15, que deduz a temperatura. $R^{\wedge} 2=0.9996$.

Figura 51-Mapa do compressor do turbo compressor ABB VTR 714, com a curva de trabalho do compressor nas faixas de carga durante os testes de aceitação.

Figura 52-Aspecto da dedução do ganho termodinâmico, pelo ajuste linear e diretamente plotado a partir dos dados de desempenho. 


\section{Lista de tabelas}

Tabela 1-Resumo dos dados de desempenho do motor do DPST

Ataulfo Alves em funcionamento de dinamômetro (Hyundai,1999). 95

Tabela 2-Momento de inércia do hélice do DPST Ataulfo Alves. 96

Tabela 3-Rendimento da turbina com aplicação de várias

faixas de carga, durante testes de aceitação em bancada. 96

Tabela 4-Rendimento do compressor com aplicação das

faixas de carga durante testes de aceitação em bancada.

Tabela 5-Consolidação dos parâmetros para cálculo da

massa de ar, obtido a partir do mapa do compressor. 


\section{Nomenclatura}

\section{Variáveis}

A - área $\left(\mathrm{m}^{2}\right)$.

A/F-razão ar combustível.

$\mathrm{a}_{\mathrm{T}}$-parâmetro de correção de fluxo.

B - consumo específico (g/kW.h).

C- ganho termodinâmico.

$\mathrm{C}_{\mathrm{v}}$-coeficiente de resistência ao escoamento de ar.

$c_{p, v}$ - calor específico à pressão e volume constante .

$\mathrm{C}_{\mathrm{T}}$ resistência ao fluxo de gás através da área efetiva média da turbina.

FPI - índice de abertura das bombas injetoras.

$\mathrm{H}, \mathrm{h}$ - entalpia .

$\mathrm{N}$ - rotação (rotação por minuto).

$\mathrm{P}$ - pressão absoluta $(\mathrm{Pa})$.

$\mathrm{J}$ - momento de inércia $\left(\mathrm{kg} \cdot \mathrm{m}^{2}\right)$.

K-constantes.

$\mathrm{kW}$ - potência $(\mathrm{kW})$

$\mathrm{m}$ - massa $(\mathrm{kg})$.

m - vazão mássica $(\mathrm{kg} / \mathrm{s})$

$\mathrm{p}$ - pressão manométrica $(\mathrm{Pa})$.

$\mathrm{PCl}$ - poder calorífico inferior $(\mathrm{MJ} / \mathrm{kg})$.

$\mathrm{Q}$ - torque. (kN.m)

$R$ - constantes do gás.

$\mathrm{R}_{\mathrm{T}}$-resistência ao avanço do casco do navio (adimensional).

$\mathrm{T}$ - temperatura $(\mathrm{K})$.

U-velocidade do navio (knots).

$\mathrm{V}$ - volume $\left(\mathrm{m}^{3}\right)$.

w-angulos de manivela (rad).

$\mathrm{W}$ - trabalho(J).

zc-número de cilindros. 


\section{Subscritos}

$a-a r$.

act-atuador.

ad- adiabático.

b-ponto de abertura da válvula de descarga.

bk-descarga do turbo compressor

c - compressão ciclo teórico.

comp - compressor.

$\mathrm{cp}$ - compressão ciclo real.

cyc- ciclo.

D- derivativo.

e-motor,efetivo.

e0-carga do motor estável.

ex-expansores fixos da turbina.

$f$ - combustível.

$\mathrm{fr}$ - fricção.

g-gás de descarga.

h-curso do motor.

i - indicado.

I-integral.

janelas - janelas de admissão de ar.

$\mathrm{L}$ - carga demandada pelo hélice.

10-carga demandada estável.

m-mecânico.

max - máximo.

mínimo - mínimo.

n-expoente politrópico.

P-proporcional.

$\mathrm{p}$ - propulsor (hélice).

q-químico.

q0-coeficiente estável do hélice.

rotor-rotor de palhetas da turbina.

s - lavagem.

$\mathrm{t}$ - térmico.

Teq-área equivalente de turbinas.

tc-turbo compressor.

tur - turbina. 
valv - válvula de descarga de gases da combustão.

z - final da combustão em ciclo teórico.

z'- ponto de início de injeção.

Veq-área equivalentes de janelas e válvulas.

2- ponto após compressor e antes do resfriador.

0-estável.

\section{$\underline{\text { Símbolos gregos }}$}

$\alpha$ - coeficiente de excesso de ar.

$\delta$-razão de expansão.

$\varepsilon$ - razão de compressão.

$\gamma$ - razão de calor específico.

$\eta$ - eficiência.

$\varphi$ - angulo de manivela (graus).

$\lambda_{c}-$ razão de pressão.

T - razão de pressão.

$\rho$ - densidade,razão de expansão preliminar.

$\tau$ - constante temporal.

$\xi$ - proporção de energia química no gás de descarga. 